\title{
Changes in normalized permutation entropy during non-coherent pulse- pulse interaction in the laser cavity
}

\author{
D. J. Little ${ }^{1}$, H. Kbashi', S. A. Kolpakov ${ }^{2 *}$, D. M. Kane ${ }^{1}$ and S. V. Sergeyev ${ }^{2}$ \\ 1. MQ Photonics Research Centre and Dept. of Physics and Astronomy, Macquarie University, Sydney, NSW 2109, Australia. \\ 2. School of Engineering and Applied Science, Aston University, Aston Triangle, Birmingham, B4 7ET, UK.
}

Statistically extreme events, that is to say, the events which have L-shaped probability distribution function (PDF, or in other words L-statistics) and, in particular, the rogue wave (RW) events, constitute a special family of natural phenomena. Curiously, this kind of events is more common than it is believed. It was observed that the behavior of the systems which obey L-shaped statistics is clustered. Periods of high predictability and low volatility are followed by periods of drastic changes and high volatility, for example, RWs [1], earthquakes, epileptic seizures etc. Permutation entropy (PE) is an indicator of dynamical predictability of the system $[2,3]$ which allows quantization of the predictability or volatility. We report an investigation of using it to characterize the uncertainty period which was observed during pulse-pulse interaction in a long ring laser resonator without a saturable absorber. These resonators, in particular, the resonator built using $\mathrm{Er}^{3+}$ doped fiber (Fig.1), have polarization dependent instability near the lasing threshold [4] which leads to the generation of pulses propagating with different speed, either randomly or regularly. Using this property, an investigator can observe the mechanism of the pulse-pulse interaction in detail.
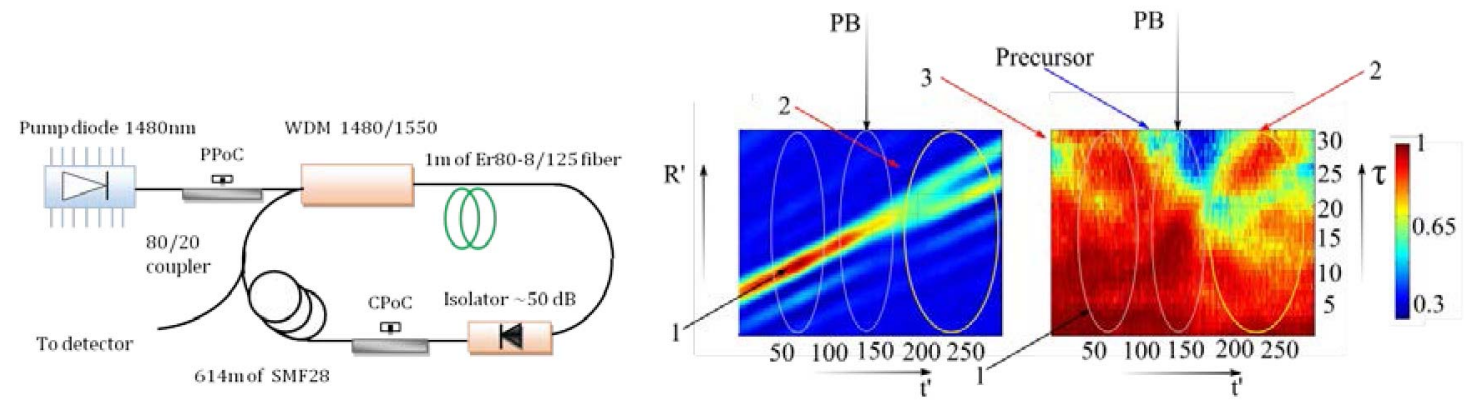

Fig. 1 Left: schematic of the laser [4]. Right: normalized PE calculated for the splitting of a lonely RW pattern. 1- lonely RW pattern, 2uncertainty period, 3- residual change in the NPE pattern due to the merging of pulses to form RW, PB- pattern breaking point.

During the experiments, it was observed that the resonance interaction between two pulses with different speed of propagation and slightly different polarization is followed by changes in the pattern of normalized permutation entropy (NPE). These changes are illustrated in Fig. 1 (right). At the beginning (1) the lonely RW pattern [4] and the precursor propagated through the laser cavity the NPE pattern is flat with the values close to 1 . The axis labeled with $R^{\prime}$ and $t^{\prime}$ symbolize roundtrips and the time during the roundtrip after Galilean transformation [4]. In this case, only the direction along R' is invariant, hence the NPE patterns were calculated along this direction $(m=4, \tau=30)$. In the moment when the patterns became unstable (2) a deep (blue color in NPE pattern). In the same moment, the precursor (the pulse before the RW) was affected presumably because of energy exchange with the main pulse. Instabilities drove the pattern to widen and flattening (the uncertainty period), which was reflected by deep blue color in NPE pattern. Finally, after the uncertainty period, when the original RW pattern was destroyed, the NPE pattern turned to be flat again (3) and small precursor appeared again before a couple of pulses. Concluding: the incoherent interaction between pulses with different polarization passes through three phases. First, the NPE pattern indicates that when internal instabilities surpass a certain level the system of pulses loses degrees of freedom and becomes more predictable; second, the pulses lose their identityforming the uncertainty pattern preceded by suppression of the high-tau component in the NPE pattern; third, a new pattern become observable and the NPE pattern become flat again.

\section{References}

[1] S. Kolpakov, H. Kbashi, S. Sergeyev, "Slow optical rogue waves in a unidirectional fiber laser," CLEO: QELS Fundamental Science, JW2A. 56, (2016).

[2] D.J. Little, D.M. Kane, "Permutation entropy of finite-length white noise time series," Phys. Rev. E. 94, 02211810 (2016).

[3] J. P. Toomey, A. Argyris, C. McMahon, D. Syvridis, D. M. Kane," Time-scale independent permutation entropy of a photonic integrated device," JLT, 19430 (2016).

[4] S.A. Kolpakov, H. Kbashi, and S V. Sergeyev, "Dynamics of vector rogue waves in a fiber laser with a ring cavity," Optica. 3, 870 (2016). 